Mots. Les langages du politique

\title{
Chantal Jaquet et Gérard Bras éd., La fabrique des
} transclasses

Paris, PUF, 2018, 279 p.

Jacques Guilhaumou

\section{OpenEdition}

\section{Journals}

Édition électronique

URL : https://journals.openedition.org/mots/24627

DOI : $10.4000 /$ mots. 24627

ISSN : 1960-6001

Éditeur

ENS Éditions

Édition imprimée

Date de publication : 5 mars 2019

Pagination : 172-176

ISBN : 979-10-362-0130-1

ISSN : 0243-6450

\section{Référence électronique}

Jacques Guilhaumou, « Chantal Jaquet et Gérard Bras éd., La fabrique des transclasses », Mots. Les langages du politique [En ligne], 119 | 2019, mis en ligne le 01 janvier 2021, consulté le 24 avril 2022 URL : http://journals.openedition.org/mots/24627 ; DOI : https://doi.org/10.4000/mots.24627

Ce document a été généré automatiquement le 24 avril 2022

(C) ENS Éditions 


\section{Chantal Jaquet et Gérard Bras éd., $L a$ fabrique des transclasses}

Paris, PUF, 2018, 279 p.

Jacques Guilhaumou

\section{RÉFÉRENCE}

Chantal Jaquet et Gérard Bras éd., La fabrique des transclasses, Paris, PUF, 2018, 279 p.

D'un auteur à l'autre de l'ouvrage se configure un Nous qui permet de comprendre ce que c'est que d'être et de se vivre transclasse. Un Nous produit d'une rencontre entre trois générations d'individus ayant effectué des trajectoires sociales inédites après la Seconde Guerre mondiale. Un Nous qui parle de l'expérience de la vie du transclasse. Un Nous qui introduit une réflexion certes inscrite dans le vécu, mais tout aussi conceptuelle. Néologisme forgé par Chantal Jaquet, auteure de Les transclasses, ou la nonreproduction (Paris, PUF, 2014), le mot transclasse fait écho à celui de class-passing utilisé dans le monde anglo-saxon. Concept axiologiquement neutre par rapport aux termes de transfuge, de parvenu ou de déclassé, il permet, selon Chantal Jaquet, d'avoir un point de vue critique sur la notion de mobilité sociale, qui met seulement l'accent sur le résultat d'une trajectoire sociale, à défaut de considérer des modes de transit particulièrement complexes. Elle critique aussi la notion de mérite, associée à l'estime, par le fait d'une logique de rétribution symbolique. Le mérite relève du seul fait de l'évaluation, donc nul ne dispose de mérite en soi. Le Nous des auteurs de cet ouvrage s'inscrit dans une telle lignée critique permettant de décrire des processus complexes de fabrication des transclasses. C. Jaquet caractérise d'emblée trois propriétés constitutives des transclasses: la déconstruction/reconstruction de l'identité personnelle, la disposition à la transgression et la conscience malheureuse. Mais la diversité des points de vue d'un auteur à l'autre de l'ouvrage introduit d'autres propriétés, ne serait-ce que du fait même de la dynamique des disciplines mises en jeu et des témoignages rassemblés. Le sociologue Paul Pasquali, auteur de Passer les 
frontières sociales (Paris, Fayard, 2014), déplace les termes liés à l'idée d'ouverture sociale, telle qu'elle est véhiculée par les élites favorables aux filières sélectives, et soucieuses de promouvoir une étroite méritocratie. Il s'agit alors de faire «quelque chose de plus ", c'est-à-dire d'observer les bénéficiaires de ces filières, à la fois par un travail de terrain centré sur le "local» et une critique des notions de "boursier" (Richard Hoggart) et de «transfuge de classe » (Pierre Bourdieu). La critique porte sur l'inadéquation de ces notions clivées, et de l'habitus qui leur est associé, avec l'observation de terrain qui montre que, en matière de migrants sociaux, ce qu'on sait de qui parle, de qui et de quoi relève de l'univers des possibles. Les résultats de l'enquête sociologique font alors écho aux récits transclassistes sous forme de témoignages. Quittant les espaces de Sciences Po, de l'Essec ou d'Henri IV, le sociologue s'intéresse à la fabrique locale de transclasses à l'initiative d'élèves d'origine populaire. Ainsi, la parole est donnée à de «jeunes migrants de classe » nullement en cours de déracinement, mais pris dans une dialectique au long cours de la frontière entre "rester soi-même » et « devenir quelqu'un d'autre ». Si le concept de "migration de classe » demeure ici opératoire, c'est par l'observation de passages perpétuels entre frontières de classe en train de se faire. La critique porte aussi sur l'opposition entre «boursiers » et « héritiers », énoncée par Albert Thibaudet en 1927, et complexifiée par P. Bourdieu. Énoncée de manière sommaire, cette opposition fait écran à la peur sociale du transfuge de classe : peur de la chute, peur de ne plus être fidèle à ses origines, aux siens, peur de la fausse note face à des collègues socialement normés. Ici domine la figure de Jules Michelet se définissant lui-même comme "homme du peuple », par souci de "rester peuple». Gérard Bras montre en quoi Michelet nous propose une généalogie de la transmigration de classes à l'époque moderne. Ici, le propre du trans est de "rester soi ». La généalogie mise en place interroge la transition du paysan à l'ouvrier, et de l'ouvrier au fabricant et son résultat, la sclérose de la bourgeoisie devenue classe. La puissance de l'esprit, associée à la puissance d'agir, disparaît au profit du devenir bourgeois. Face à l'envie, il s'agit pour Michelet de maintenir une éthique du "rester soi » qui permet de s'estimer soi-même. La voie vers l'ascension sociale, c'est alors le travail permettant de disposer des "deux sexes de l'esprit », donc de se tenir sur les deux bords de la fracture sociale franchie, actualisée dans le passage du jeune Michelet typographe composant des livres au Michelet historien universitaire, qui écrit des livres. Les deux sexes de l'esprit se conjoignent dans «l'homme de génie ", apte à cerner « la personnalité du peuple » dans les termes mêmes de Michelet : « cette personnalité [...] je ne l'ai point vue du dehors, mais expérimentée du dedans». Par contraste, "le portrait du pur littérateur en déclassé volontaire " au XIX $x^{e}$ siècle, présenté par Ronan de Calan, situe des hommes de lettres déclassés volontaires, renonçant à l'avant-gardisme politique après les massacres de juin 1848, et revendiquant un certain ethos aristocratique. Théophile Gautier écrit ainsi qu'« il y aura toujours parmi les hommes une aristocratie que nulle république ne supprimera, celle des poètes ", en écho à Charles Baudelaire célébrant le poète "aristocrate de nature plus encore que de naissance » avec la figure d'Edgar Allan Poe. Le "pur littérateur » est pris dans la crise identitaire du transclasse du fait de sa souffrance dans l'écriture, et donc de sa rareté, concrétisée à l'extrême par l'absence d'œuvre d'une part, et du fait d'un travail d'autocensure suscité par une mauvaise conscience «bourgeoise » d'autre part.

2 Les témoignages singuliers proposés, sous forme de récits réflexifs, constituent autant de postes d'observation à valeur critique. Si les sentiments de peur et de honte sont 
présents dans le parcours transclasse, il y est aussi question d'écriture salvatrice, de maitrise permanente de soi-même. Le récit de "passe-classe " proposé par Martine Sonnet, historienne des sociabilités éducatives et culturelles, part d'un vécu spécifique de son trajet transclasse avec en son centre le lien entre écriture et parcours social. Paysan d'origine, son père est ouvrier à l'usine de Boulogne-Billancourt. Fille d'ouvrier, elle ne se présente pas comme telle dans son milieu professionnel, jusqu'à la publication d'Atelier 62, qui relate la trajectoire familiale et professionnelle de son père. Ici, l'écriture à la première personne, tant attendue et enfin réalisée, rend lisible ce qui précède, soit un parcours transclasse vertical vers le métier d'ingénieur de recherche au CNRS après des années de précarité, en y associant le parcours transclasse latéral de son père, artisan campagnard ouvriérisé. Pour sa part, Patricia Janody, psychanalyste, propose le récit de «l'expérience d'un franchissement de seuil qui était répétitivement celui de la honte », processus riche de potentialités au sens transclassiste. À la psychose de la mère, puis du frère, source d'enfermement dans le huis clos familial, se surimpose la honte d'être sous le regard du libraire et d'une clientèle avertie lorsqu'elle entre dans une librairie, alors que la lecture était l'espace principal d'échappement au poids familial, grâce à la fréquentation de la bibliothèque municipale. P. Janody perçoit un sentiment de honte qui s'exprime dans les termes d'une circulation trouble entre son corps propre et l'objet livre, dans la mesure où ce sentiment de ne-pas-savoir-lire perdure en elle et induit un mode de lecture immanent, donc hors des discours savants. Et d'en conclure paradoxalement : «La honte me socialisait », dans la mesure où elle lui permet de se rattacher à une instance tierce centrée sur un autre « hypothétique » au sein d'un collectif. Dans le trajet de P. Janody se précisent, en termes transclassistes, non seulement un passage réalisé de classe, mais aussi une construction "hypothétique " à la jointure de l'individuel et du social, susceptible d'aboutir à la participation au présent ouvrage collectif. Pour sa part, Jean-Louis Saporito met l'accent sur une enfance heureuse dans une famille où le père est petit patron coiffeur, et la mère secrétaire de direction à Paris, au sein d'un quartier populaire, le bas de Saint-Cloud, en constante confrontation avec le quartier bourgeois le plus proche, le haut de Saint-Cloud. Dans un quartier où vivaient surtout des familles ouvrières, J.L. Saporito devient différent par son aptitude à poursuivre ses études jusqu'au lycée technique. S'il répond à l'ambition des parents par le mérite personnel, un sentiment d'imposture saisit l'adolescent et il en ressort la peur constante de l'échec. À se construire une vie différente de ses parents, il ne dispose plus que de l'usage de l'improvisation dans une vie où il faut tout inventer, de la carrière de journaliste à la profession de PDG d'une agence de presse. Le trajet de Patrick Bourdet relève inversement d'une enfance difficile, marquée par une vie misérable dans une cabane au cœur de la forêt, sans aucun confort, au côté d'une mère alcoolique. À 16 ans, P. Bourdet s'enfuit, fait appel à la DDASS et se retrouve dans une famille d'accueil vivant dans de bonnes conditions. Disposant d'un CAP de mécanique, il devient ouvrier contremaître après plusieurs années d'activité professionnelle tout en continuant ses études jusqu'à obtenir le baccalauréat. Décrocher des diplômes, accumuler les formations ne cessera de lui procurer un sentiment de protection. Enchaînant les métiers de technicien, technicien supérieur, agent administratif, cadre, ingénieur, cadre supérieur, il devient PDG d'une société spécialisée dans le développement d'anticancéreux, puis d'une société de conseil, tout en suivant un cursus de Gestaltthérapeute. Il perçoit la richesse de son parcours dans sa propre histoire, et la maîtrise qu'elle lui impose personnellement. Pour sa part Annie Tardits interroge le paradoxe 
d'être transclasse dans un lieu transclasse, l'école normale d'instituteurs. Excellente élève issue d'un milieu social de petits paysans, petits artisans et petits commerçants, elle est orientée vers un cycle long d'études classiques. Au collège, elle éprouve un sentiment tenace de honte face aux élèves de familles aisées. Enseignante à l'école normale d'instituteurs, elle se demande si la figure du transclasse peut s'insérer dans la visée d'un changement politique et syndical. À ce titre, elle s'interroge sur la portée transclassiste des mutations de l'école normale d'instituteurs. Elle est consciente que ces mutations sont prises en charge par des professeurs qui ne sont pas des "héritiers", qui sont donc de "couleur transclasse». Elle participe à un collectif militant soucieux d'inventer de nouveaux dispositifs pédagogiques en lien avec une réflexion sur le savoir. Certes, elle reprend des études de philosophie à l'École normale supérieure de Fontenay-aux-Roses et à la Sorbonne et devient psychanalyste. Mais elle garde une sensibilité propre héritée de sa vie d'institutrice et de son implication politique durant cette période.

3 Le questionnement des contributions à cet ouvrage se précise par l'apport conjoint d'une confrontation du concept de transclasse avec la question de la langue et du bilinguisme d'une part, et de la portée du rapport conceptuel classe/transclasse d'autre part. Le récit de Soubattra Danasségarane s'inscrit dans une interrogation critique spécifique: qu'en est-il de l'identité des langues dans le contexte bilingue d'une Française d'origine immigrée? De parents d'origine tamoule, S. Danasségarane est née en France, mais a passé une partie de son enfance en Inde. Le français est « sa langue ", mais elle ne la ressent pas comme totalement légitime, jusque dans sa version philosophique, dont elle est dépositaire en tant que professeure agrégée de philosophie. Elle est tiraillée par toutes sortes de conflits au sein de sa «langue officielle » du fait qu' « elle subit plusieurs champs sémantiques ». Habiter une langue s'avère paradoxal : il faut se légitimer en permanence, même au plus haut niveau de sa connaissance de la langue; il faut se déprendre de l'idée qu'une identité acquise renvoie d'emblée à une maîtrise de la langue apprise. Ainsi, dans le jeu complexe des conflits identitaires, la confrontation des langues parlées est centrale. Le tamoul est ici la langue de l'affect, de l'intime et de la filiation, de la mémoire, au détriment d'une maîtrise écrite jamais vraiment atteinte; le français est la langue qu'il fallait parler, tant dans les tâches pratiques que dans l'échange intellectuel, il est ce qui permet l'accès au savoir, mais aussi à la liberté, il incarne la liberté, et l'émancipation dans le genre. Mais la crise identitaire reste présente en matière de langue par le fait du partage entre la perception d'une maîtrise toujours insuffisante du tamoul et l'usage d'un français parfois clivant, donc source de honte. Ainsi en est-il de l'épisode où elle oublie le concept d'habitus à un oral de khâgne, ce qui lui vaut du professeur une question sur sa langue maternelle. Il s'agit bien ici de rendre compte d'une expérience identitaire située aux limites de l'intraduisible au sein d'un bilinguisme immigré transclasse, du fait de l'unicité de chaque langue et de chaque culture. Qu'en est-il alors du concept de transclasse par rapport à celui de classe ? Vincent Houillon considère les usages du terme déconstruction chez $\mathrm{C}$. Jaquet pour qualifier la complexité des catégories nécessaires à la compréhension du phénomène de transclasse, au regard des logiques de l'écart, de l'espacement, puis de l'éloignement. Il propose d'en situer le prolongement au sein de la dynamique de classe. Il précise ainsi l'apport de Jacques Derrida en matière d'interpellation du transclasse, sous la forme du "peut-être » de l'appartenance de classe par lequel toute substantialité de l'identité est suspendue. V. Houillon en vient à caractériser un espace de non-identité de la classe à soi, de 
l'autodéconstruction traversée par le transclasse qui n'est rien d'autre que sa propre traversée, son trajet à travers les classes. Parler de trans revient alors à situer une dynamique et sa puissance de virtualité, à qualifier une expression de classe pouvant virtuellement advenir. Ainsi le transclasse ressent l'inadéquation de toute classe à soi, à son propre ou à son identité, tout en continuant à dénoncer la domination de classe.

\section{AUTEURS}

JACQUES GUILHAUMOU

ENS de Lyon, Triangle 